\title{
Vol. 68, No. 43
}

In the report "Update: Characteristics of Patients in a National Outbreak of E-cigarette, or Vaping, Product UseAssociated Lung Injuries — United States, October 2019," on page 989, the list of contributors on the Lung Injury Response Epidemiology/Surveillance Task Force should have included Samantha J. Lange, National Center for Chronic Disease Prevention and Health Promotion, CDC 